\title{
Protein-Losing Gastroenteropathy as Cause of Hypoproteinemia in Constrictive Pericarditis
}

\author{
Shigekoto Kaimara, M.D.,* Hiroko Nishimura, M.D.,* \\ Toshio Ajyagi, M.D., ${ }^{*}$ Haruo Kameda, M.D.,* \\ and Hideo Ueda, M.D.*
}

$\mathrm{H}^{\mathrm{x}}$

YPOPROTEINEMIA has been reported to be associated with some cases of cardiac diseases and it has been studied with much interest especially in the similarity to liver cirrhosis. Many hypotheses have been proposed for the explanation of its mechanism, ${ }^{12), 13)}$ but they had been unable to get general acceptance, until the gastrointestinal protein loss was measured with $\mathrm{I}^{131}$-labelled polyvinylpyrrolidone ( ${ }^{131}$-P.V.P.) in these cases. ${ }^{1)-3)}$ This method was first devised for the investigation of hypoproteinemia in gastrointestinal diseases and essential hypoproteinemia, but Davidson et al. ${ }^{14}$ ) applied it to cardiac diseases with hypoproteinemia and revealed that the same mechanism was responsible for the development of hypoproteinemia in cardiac diseases.

Recently, we experienced a case of constrictive pericarditis with hypoproteinemia, in which performing the $I^{131}-P . V . P$. test, we could show the abnormal gastrointestinal protein loss. Moreover, the biopsy of the intestinal mucosa was carried out to reveal the dilatation of lymphatic vessels, that was identical with the findings in essential hypoproteinemia reported by Waldmann et al. ${ }^{18)}$ as intestinal lymphangiectasia.

\section{GASE R $\mathrm{R}_{\text {EPORT }}$}

This Japanese house-wife was first admitted in July, 1962, at the age of 41 , for the investigation of edema of over 4 years' duration. Except for the usual infectious diseases of childhood, she had been in good health until May, 1957, when she noted exertional pain of the left knee-joint with the feeling of dullness of the left leg. Shortly afterwards, the joint swelled with the evidence of local inflammation. She, however, did not have any systemic difficulties at that time. In May, 1958, the pain of the left knee-joint started again and she developed fever to as high as $40^{\circ} \mathrm{C}\left(104^{\circ} \mathrm{F}\right)$. She noted the gradual onset of edema involving the ankles and the legs. Under the diagnosis of tuberculous arthritis of the left knee-joint, she was treated with drainage and plaster and 1 year afterwards

* From the Second Department of Internal Medicine, Faculty of Medicine, University of Tokyo, Tokyo. 
it healed leaving arthrodesis of the joint. But her edema had become more remarkable involving all over the body, especially the left ankle and leg, where edema was always the most severe. She started having slight exertional dyspnea and dull chest pain. In July 1962, her edema became refractory and increased in severity; she was admitted to the Tokyo University Hospital for the evaluation of her edema.

She was a well-developed, well-nourished Japanese female not in acute distress. The salient feature was pitting edema of the face, abdominal wall and the lower extremities. The edema of the lower extremities was asymmetric with the left more remarkable than the right. There was no venous engorgement in the neck. The blood pressure was $120 / 84 \mathrm{~mm} . \mathrm{Hg}$ and the pulse rate was 82 , regular and not paradoxical. There was no evidence of cardiac enlargement on percussion and protodiastolic extrasound was audible on auscultation to the left of the sternum in the fourth left interspace. Abdomen was distended with accumulation of ascites. Liver, spleen and kidneys were not palpable. The left knee-joint was fixed in 170 degree without any evidence of acute inflammation.

Venous pressure was $210 \mathrm{~mm}$. saline and circulation time was 18 sec. (arm-to-tongue time) and $14 \mathrm{sec}$. (arm-to-lung time). Routine blood-counts and urinalysis were normal. The serum-protein level was $4.2 \mathrm{Gm}$. per $100 \mathrm{ml}$. with $1.9 \mathrm{Gm}$. albumin. Serum calcium was $8.1 \mathrm{mg}$. per $100 \mathrm{ml}$. The following determinations and tests gave results within normal limits: blood-glucose, bloodurea, cholesterol, electrolytes, bilirubin, thymol-turbidity test, zinc-sulphate test, alkaline phosphatase and serological test for syphilis. Bromsalphalein retention was $20 \%$ after $45 \mathrm{~min}$. on admission, but shortly afterwards it returned to normal. Ascites was transudate in nature and chylous in outlook. X-ray examination of the chest showed normal contour of the heart both in size and shape; calcification of the pericardium was not present (Fig. 1). The electrocardiogram showed low voltage of $Q R S$ complexes and inversion of $\mathrm{T}$ waves in chest leads (Fig. 2). Kymographic examination revealed almost normal pulsation of

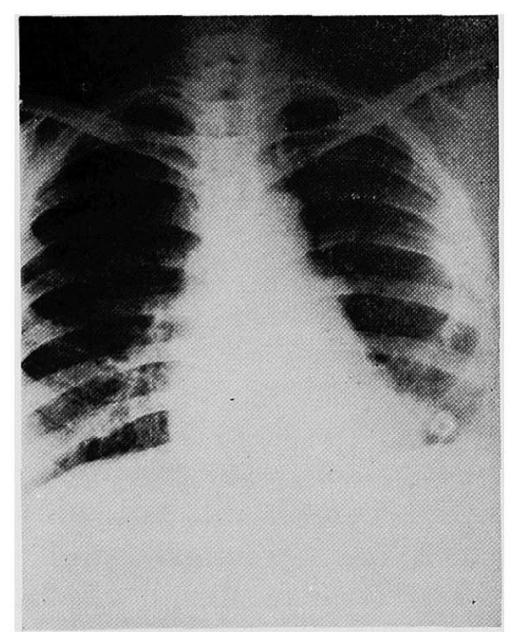

Fig. 1. Chest X-ray film, showing normal sized heart. 


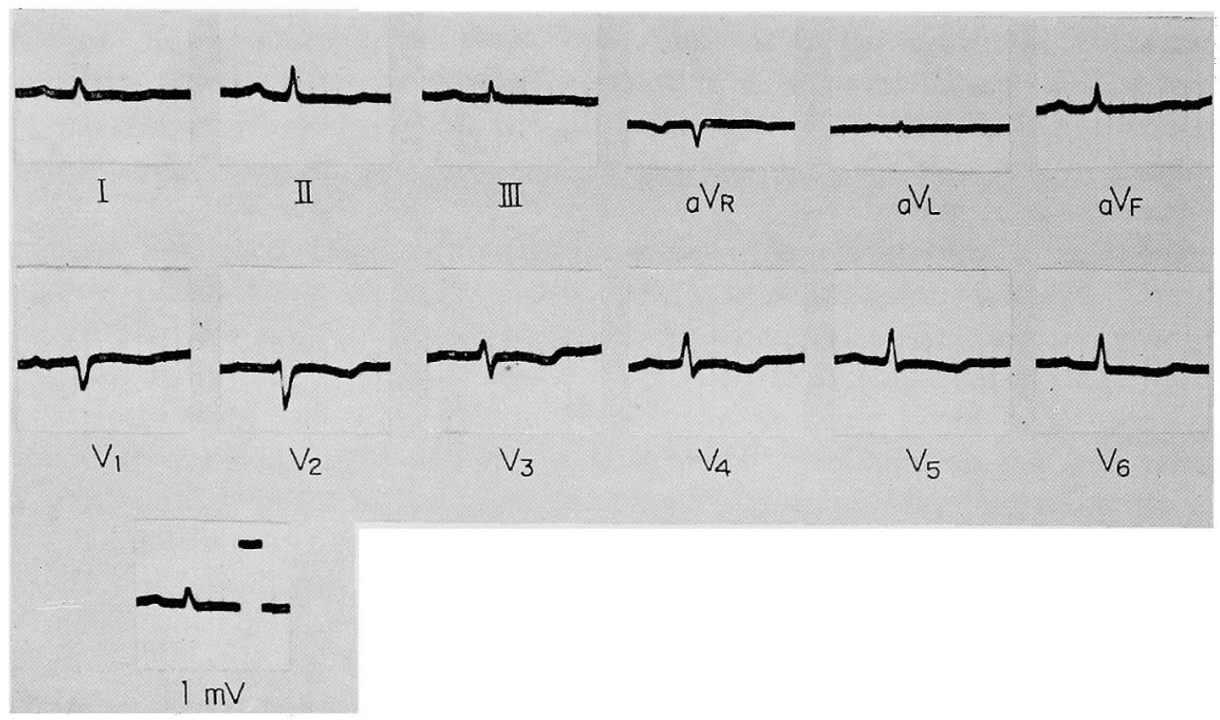

Fig. 2. Electrocardiogram.

the heart. Phonocardiogram showed that protodiastolic extrasound was 0.08 sec. distant from the aortic component of the second sound, which was characteristic of constrictive pericarditis (Fig. 3). Transseptal left heart catheriza-

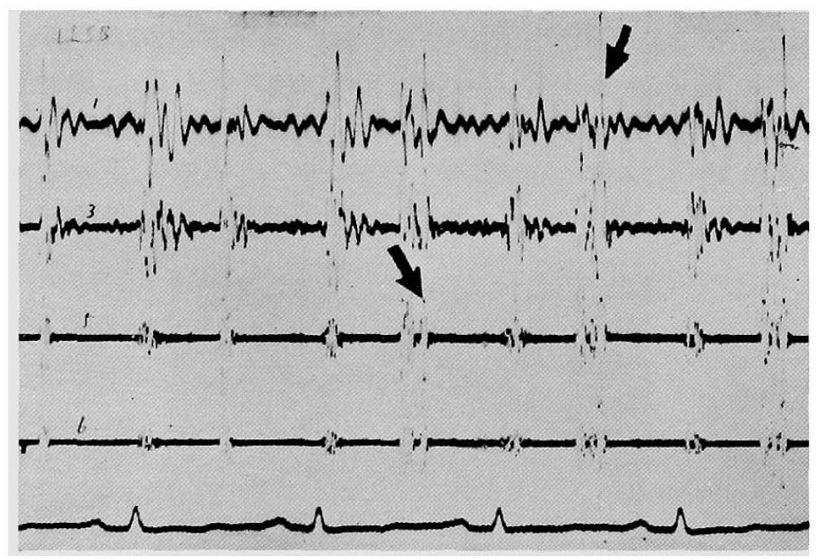

Fig. 3. Phonocardiogram at the left of the sternum in the fourth interspace, showing protodiastolic extrasound.

tion and right heart catheterization were performed with the demonstration of elevated atrial pressures and diastolic dip and plateau in the right ventricular pressure curve (Fig. 4), which were compatible with the diagnosis of chronic constrictive pericarditis. The end-diastolic to systolic pressure ratio(1) of the ventricle was 54\%. Study of the gastrointestinal tract with barium showed no abnormalities. Laparoscopic examination showed congestive liver. 


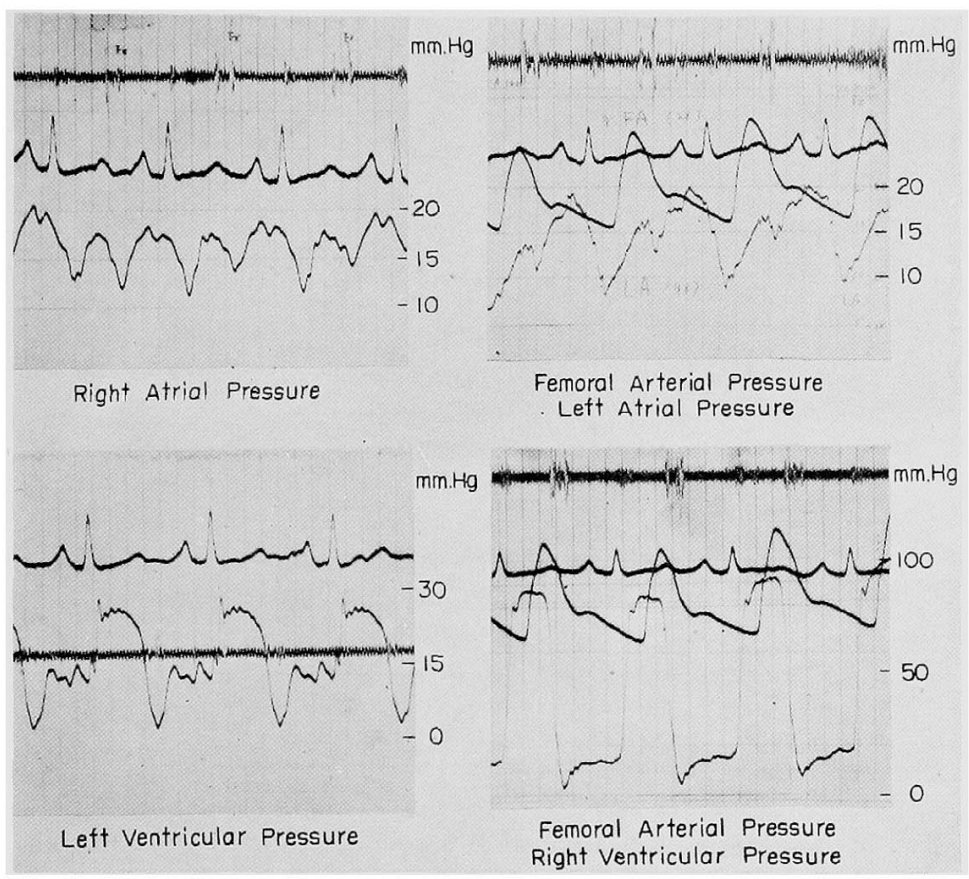

\begin{tabular}{c|c|c}
\hline & Right Atrial Pressure & Left Atrial Pressure \\
\hline $\mathrm{a}$ & $18.5 \mathrm{~mm} \cdot \mathrm{Hg}$ & $22.5 \mathrm{~mm} . \mathrm{Hg}$ \\
$\mathrm{v}$ & $16.5 \mathrm{~mm} \cdot \mathrm{Hg}$ & $17.0 \mathrm{~mm} . \mathrm{Hg}$ \\
$\mathrm{m}$ & $15.0 \mathrm{~mm} \cdot \mathrm{Hg}$ & $17.0 \mathrm{~mm} . \mathrm{Hg}$ \\
\hline \hline & Right Ventricular Pressure & Left Ventricular Pressure \\
\hline Systolic Pressure & $27.0 \mathrm{~mm} \cdot \mathrm{Hg}$ & $94.0 \mathrm{~mm} . \mathrm{Hg}$ \\
Diastolic Pressure & $14.5 \mathrm{~mm} \cdot \mathrm{Hg}$ & $20.0 \mathrm{~mm} . \mathrm{Hg}$ \\
Wave Pattern & dip and plateau & \\
\hline
\end{tabular}

Fig. 4. Cardiac catheterization, showing the curve characteristic of constrictive pericarditis in right ventricular pressure curve.

To investigate the mechanism of hypoproteinemia, abnormal gastrointestinal permeability to macromolecules was examined with I $^{131}$-labelled polyvinylpyrrolidone*. This was carried out after the procedure described by Gordon, ${ }^{13}$ and indicated the abnormal result, that fecal excretion of $I^{131}-P . V . P$. for 4 days was $7.33 \%$ in this case while it was $1.12 \%$ in control (Fig. 5).

Albumin turnover was measured with I $^{131}$-labelled human serum-albumin*. After thyroidal uptake of $\mathrm{I}^{131}$ had been blocked by the administration of stable iodine, the degradation of the labelled albumin was followed by measurement of serum radioactivity and the rate of urinary excretion.4) From these data

* obtained from Abbott Laboratories. 


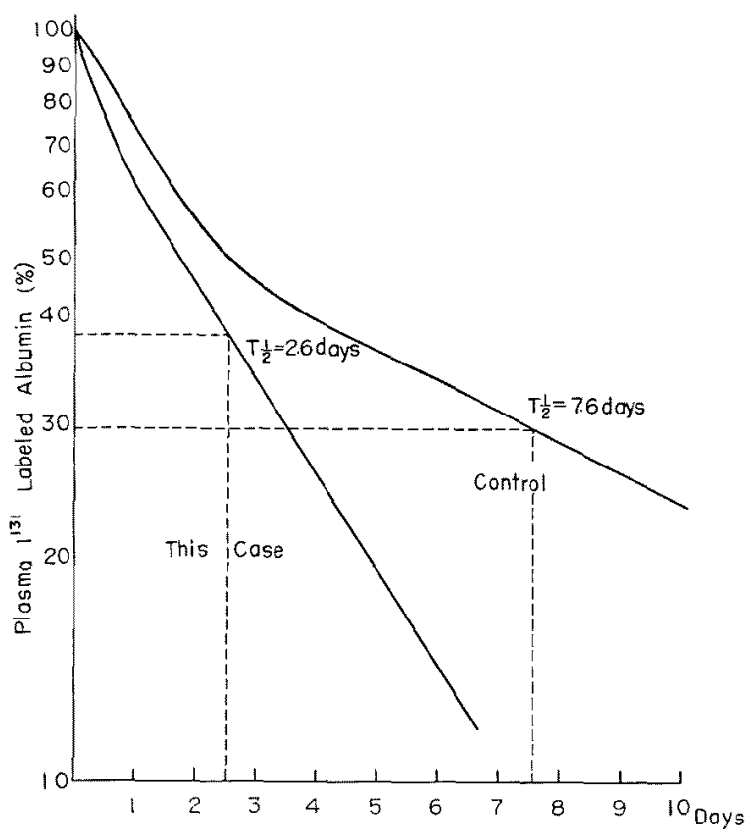

Fig. 5. Disappearance curve of $\mathbf{I}^{131}$-labelled albumin from plasma.

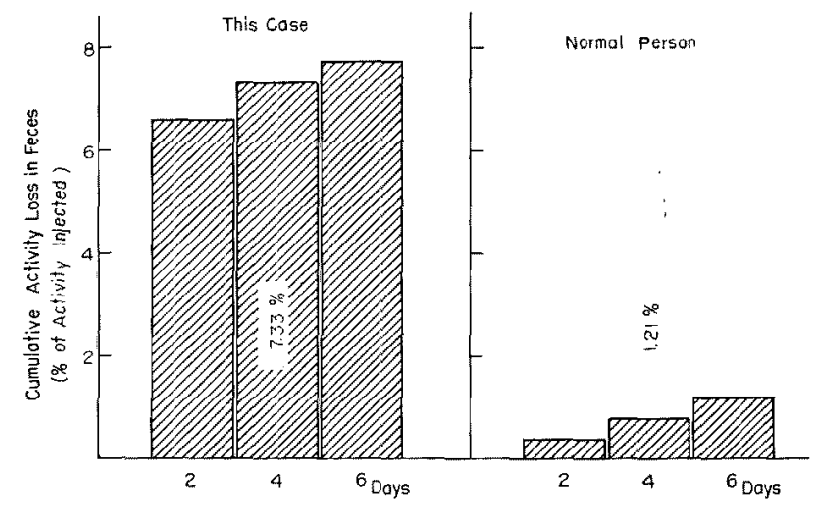

Fig. 6. I'131-P.V.P. excretion in feces in this case and normal person.

half-time for albumin degradation and absolute rate of albumin turnover could be calculated (Fig. 6). These were 2.6 days and $0.23 \mathrm{Gm} . / \mathrm{Kg} . /$ day in this case and 7.2 days and $0.14 \mathrm{Gm} . / \mathrm{Kg}$./day in control. These studies indicate hypoproteinemia is the result of an exaggerated rate of albumin catabolism, but not of a failure of albumin synthesis.

Peroral suction biopsy of the intestinal mucosa by Crosby tube was carried out with the demonstration of a variable degree of dilatation of the lymphatic vessels of the intestinal mucosa, identical with the findings reported by Waldmann et al. as intestinal lymphangiectasia in some cases of essential hypoproteinemia (Fig. 7 and 8 ). 

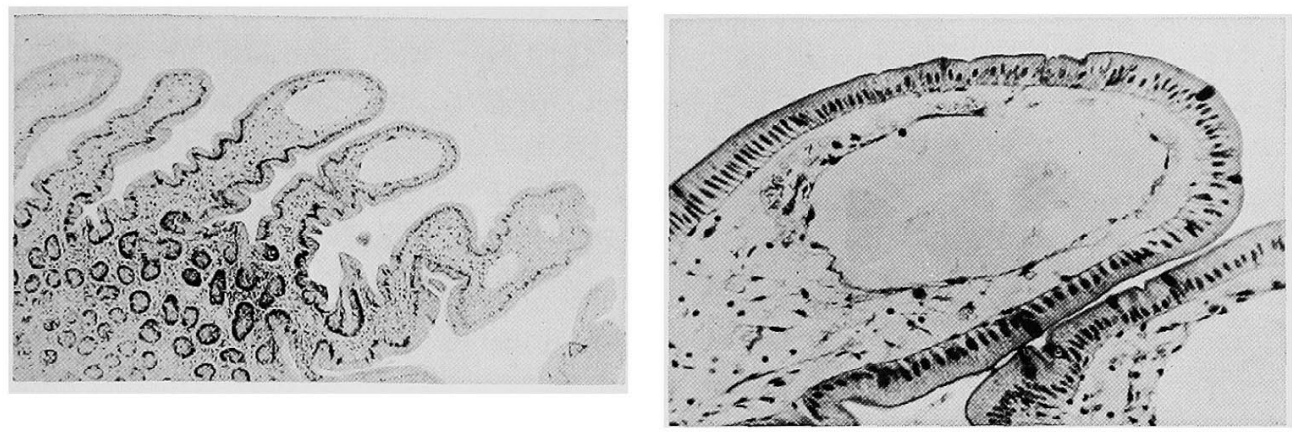

Fig. 7-8. Jejunal biopsy, showing dilatation of mucosal lymphatic vessels. (H \& E)

On a programme of bed rest, salt restriction and diuretics the patient had a good diuresis for some time with marked weight loss, but serum protein level did not become over $5.0 \mathrm{Gm}$. per $100 \mathrm{ml}$. with reappearance of edema shortly afterwards. She did not get well enough to tolerate operations.

\section{Discussion}

Though there remain some points that are not typical, protodiastolic extrasounds in phonocardiogram, low voltage in electrocardiogram with inversion of $\mathrm{T}$ wave and dip and plateau in the right ventricular pressure curve observed in this case, indicate constrictive pericarditis as its diagnosis. Though the calcification of the pericardium was not found in this case, it is reported to be shown in only $50 \%$ of the cases. ${ }^{5)}$ Diminished cardiac movements are not the constant findings for constrictive pericarditis. ${ }^{6}$ ) It has been reported that similar pressure curves in cardiac catheterization are encountered in cases with right ventricular failure, myocardial fibrosis or myocarditis of obscure origin. $\left.{ }^{77}, 87,199,20\right)$ But in constrictive pericarditis the end-diastolic to systolic pressure ratio is reported to exceed one third, ${ }^{11)}$ which was $54 \%$ in this case.

Hypoproteinemia has been reported in association with constrictive pericarditis. Its incidence is variable ranging from $20 \%$ to $50 \%$ according to the reporters. In our clinic hypoproteinemia less than $6.0 \mathrm{Gm}$. per $100 \mathrm{ml}$. has been encountered in about one third of the patients with constrictive pericarditis. To explain the mechanism of this hypoproteinemia, many hypotheses have been proposed. ${ }^{12), 13)}$ 1) Impaired synthesis of serum protein due to malnutrition. 2) Dilution of serum protein due to the increase of plasma volume. 3) Exaggerated loss of serum protein through peritoneal punctures. 4) Impaired protein synthesis due to congestive liver. 5) Degradation of serum protein by some unknown mechanism. But neither of them could give sufficient explanations for the mechanism of hypoproteinemia. In recent years, however, Gordon ${ }^{1)}$ 
reported a method to measure gastrointestinal leakage of serum protein using $\mathbf{I}^{131}-\mathrm{P}$. V.P. Davidson et $\mathrm{al}^{14)}$ applied this method to cardiac diseases with hypoproteinemia and revcaled abnormal gastrointestinal protein loss. After hypoproteinemia improved by the surgical removal of the pericardium, he examined this again and found the gastrointestinal leakage of serum protein was not present any more in successful postoperative cases. Consequently, he concluded this gastrointestinal leakage of serum protein was the cause of hypoproteinemia in cardiac diseases.

In this case liver functions remained within normal limits and albumin turn-over studies showed the synthesis of serum albumin was not impaired but increased more than normal. There was no primary diseases of the gastrointestinal tract. These facts indicate the gastrointestinal protein loss in this case was related to cardiac disease itself; consequently this is the identical case with the ones of Davidson et al.

Table I gives the cases of cardiac diseases in which gastrointestinal protcin loss was reported.

Table I. Reports of Excessive Gastrointestinal Loss of Plasma Protein in Patients with Gardiac Diseases

\begin{tabular}{|c|c|c|c|}
\hline & $\begin{array}{c}\text { Total } \\
\text { Protein } \\
\mathrm{Gm} . / 100 \mathrm{ml} .\end{array}$ & $\begin{array}{c}\text { Albumin } \\
\mathrm{Gm} . / 100 \mathrm{ml}\end{array}$ & $\underset{\%}{\text { P.V.P. Test }}$ \\
\hline \multicolumn{4}{|l|}{ Davidson et al.14 } \\
\hline 1. Constrictive Pericarditis & 3.1 & 1.6 & 6.9 \\
\hline 2. Constrictive Pericarditis & 3.7 & 1.5 & 5.3 \\
\hline 3. Atrial Septal Defect & 3.7 & 2.2 & 92 \\
\hline 4. Constrictive Pericarditis & 4.7 & 2.0 & 5.9 \\
\hline \multicolumn{4}{|l|}{ Hoedt et al.16) } \\
\hline 1. Constrictive Pericarditis & & 4.1 & 0.38 \\
\hline 2. Constrictive Pericarditis & & 1.6 & 5.27 \\
\hline 3. Constrictive Pericarditis & & 1.3 & 9.23 \\
\hline \multicolumn{4}{|l|}{ Jeejeebhoy ${ }^{157} \quad$ (1962) } \\
\hline 1. Constrictive Pericarditis & & 2.7 & $7.5 *$ \\
\hline 2. Pulmanary Stenosis & & 1.7 & $6.0 *$ \\
\hline \multicolumn{4}{|l|}{ Oeff et al. ${ }^{213}$} \\
\hline Constrictive Pericarditis & 4.0 & 1.7 & 8.9 \\
\hline Tanoue $\quad$ (1962) & & & \\
\hline Constrictive Pericarditis & 3.8 & 1.7 & 7.75 \\
\hline
\end{tabular}

What is the mechanism of gastrointestinal protein loss, then? In the study of "essential hypoproteinemia", Waldmann et al. ${ }^{18}$ ) revealed the increased gastrointestinal plasma protein loss and on the basis of patho- 
histological study of their intestinal mucosa, that were mostly obtained by suction biopsy, he found a variable degree of dilation of the lymphatic vessels of the intestinal mucosa. He named it intestinal lymphangiectasia. Together with the clinical manifestations of the cases that pleural or ascitic effusion was chylous, and that the edema on lower extremities was asymmetric, he attributed the mechanism of the gastrointestinal protein loss in essential hypoproteinemia to a disorder of the lymphatic system of the small bowel.

It is likely that the operative mechanism in patients with cardiac diseases is also this lymphatic disorder of the small bowel. But as far as we know, there has been no report on the findings of the intestinal biopsy in patients with cardiac diseases. The intestinal biopsy of our case, however, revealed the dilatation of the lymphatic vessels of the intestinal mucosa, which was identical with the findings of Waldmann's cases. This case had a similarity to Waldmann's cases also in clinical manifestations, that ascitic effusion was chylous and that edema on lower extremities was asymmetric. We think these facts are good evidences for that, for the appearance of hypoproteinemia the operative mechanism is the same in cardiac diseases as in essential hypoproteinemia.

\section{Summary}

A case of constrictive pericarditis with hypoproteinemia was reported with the demonstration of gastrointestinal protein loss and lymphangiectasia of the intestinal mucosa. From these facts hypoproteinemia in cardiac diseases was revealed to be due to gastrointestinal protein loss caused by a disorder of the lymphatic vessels of the small bowel.

\section{ADDENDUM}

The patient died on 2 July, 1963. Autopsy was performed with the findings of fibrous thickening of the pericardium constricting the heart, and the sequela of pleurisy, which were considered to be tuberculous in origin.

\section{REFERENCE}

1. Gordon, R.S.: Lancet 1 : 325, 1959.

2. Jeffries, G.H., et al.: New Engl. J. Med. 266: 652, 1962.

3. Bennhold, V.H. and Ott, H. : Med. Klin. 57: 814, 1962.

4. Veal, N. and Vetter, H.: Radioisotope techniques in clinical research and medicine: Butterworth, 1958.

5. Steinberg, I. : Am. J. Cardiol. 7: 33, 1961.

6. Heinz, R, and Abrans, H.L. : Radiology 69 : 54, 1957. 
7. Hansen, A.T. et al.: Circulation 3:881, 1951.

8. McKusick, V.A.: Bull. Johns Hopkins Hosp. 90 : 3, 1952.

9. Wood, P.: Am. J. Cardiol. 7: 48, 1961.

10. Eliot, E.S., et al.: Circulation 23: 613, 1961.

11. Yu, P.N.G., et al.: Circulation 7: 102, 1953.

12. Stadler, H. and Stinger, D.: J. Pediat. $18: 84,1941$.

13. Moschcowitz, E.: Ann. Int. Med. 36: 933, 1952.

14. Davidson J.D., et al. : Lancet 1: 899, 1961.

15. Jeejeebhoy, K.N.: Lancet $1: 343,1962$.

16. Hoedt, K., et al. : Lancet 1: 1110, 1961.

17. Schwartz, M. and Jarnum, S.: Lancet 1: 327, 1959.

18. Waldmann, T.A., et al.: Gastroenterology 41: 197, 1961.

19. Von Hilger, J.H., et al.: Arch. Kreislaufforsch. 38: 260, 1962.

20. Von Rudolph, W. and Bernsmeier, A.: Z. Kreislaufforsch. 51: 52, 1962.

21. Oeff, K., et al.: Nuclearmedizin 2: 234, 1962. 